\title{
Pd 催化甲醇裂解制氢的反应机理
}

\author{
倪哲明 * 毛江洪潘国祥胥倩李小年 \\ (浙江工业大学化学工程与材料学院, 杭州 310032)
}

\begin{abstract}
摘要：基于密度泛函理论(DFT), 研究了甲醇在 $\mathrm{Pd}(111)$ 面上首先发生 $\mathrm{O}-\mathrm{H}$ 键断裂的反应历程 $\left(\mathrm{CH}_{3} \mathrm{OH}(\mathrm{s}) \rightarrow\right.$ $\left.\mathrm{CH}_{3} \mathrm{O}(\mathrm{s})+\mathrm{H}(\mathrm{s}) \rightarrow \mathrm{CH}_{2} \mathrm{O}(\mathrm{s})+2 \mathrm{H}(\mathrm{s}) \rightarrow \mathrm{CHO}(\mathrm{s})+3 \mathrm{H}(\mathrm{s}) \rightarrow \mathrm{CO}(\mathrm{s})+4 \mathrm{H}(\mathrm{s})\right)$. 优化了裂解过程中各反应物、中间体、过渡态 和产物的几何构型, 获得了反应路径上各物种的吸附能及各基元反应的活化能数据. 另外, 对甲醇发生 $\mathrm{C}-\mathrm{O}$ 键断裂生成 $\mathrm{CH}_{3}(\mathrm{~s})$ 和 $\mathrm{OH}(\mathrm{s})$ 的分解过程也进行了模拟计算. 计算结果表明, $\mathrm{O}-\mathrm{H}$ 键的断裂(活化能为 103.1 $\mathrm{kJ} \cdot \mathrm{mol}^{-1}$ ) 比 $\mathrm{C}-\mathrm{O}$ 键的断裂(活化能为 $249.3 \mathrm{~kJ} \cdot \mathrm{mol}^{-1}$ ) 更容易; 甲醇在 $\mathrm{Pd}(111$ )面上裂解的主要反应历程是: 甲 醇首先发生 $\mathrm{O}-\mathrm{H}$ 键的断裂, 生成甲氧基中间体 $\left(\mathrm{CH}_{3} \mathrm{O}(\mathrm{s})\right)$, 然后甲氧基中间体再逐步脱氢生成 $\mathrm{CO}(\mathrm{s})$ 和 $\mathrm{H}(\mathrm{s})$. 甲醇发生 $\mathrm{O}-\mathrm{H}$ 键断裂的活化能为 $103.1 \mathrm{~kJ} \cdot \mathrm{mol}^{-1}$, 甲氧基上脱氢的活化能为 $106.7 \mathrm{~kJ} \cdot \mathrm{mol}^{-1}$, 两者均有可能是 整个裂解反应的速控步骤.
\end{abstract}

关键词：甲醇裂解； 反应机理；密度泛函理论；过渡态

中图分类号：0641

\section{Mechanism of Palladium-Catalyzed Methanol Decomposition for Hydrogen Production}

\author{
NI Zhe-Ming* MAO Jiang-Hong PAN Guo-Xiang XU Qian $\quad$ LI Xiao-Nian \\ (College of Chemical Engineering and Materials Science, Zhejiang University of Technology, Hangzhou 310032, P. R. China)
}

\begin{abstract}
The reaction pathway of methanol decomposition $\left(\mathrm{CH}_{3} \mathrm{OH}(\mathrm{s}) \rightarrow \mathrm{CH}_{3} \mathrm{O}(\mathrm{s})+\mathrm{H}(\mathrm{s}) \rightarrow \mathrm{CH}_{2} \mathrm{O}(\mathrm{s})+2 \mathrm{H}(\mathrm{s}) \rightarrow \mathrm{CHO}\right.$ $(\mathrm{s})+3 \mathrm{H}(\mathrm{s}) \rightarrow \mathrm{CO}(\mathrm{s})+4 \mathrm{H}(\mathrm{s}))$ on $\mathrm{Pd}(111)$ surfaces was studied using density functional theory (DFT). Geometries of reactants, intermediates, transition states and products were calculated. Adsorption energies of possible species and activation energy barriers of possible elementary reactions involved in the mechanism were obtained in this work. In addition, we studied the reaction mechanism for $\mathrm{C}-\mathrm{O}$ bond scission in methanol decomposition, which led to the formation of $\mathrm{CH}_{3}(\mathrm{~s})$ and $\mathrm{OH}(\mathrm{s})$. Results show that $\mathrm{O}-\mathrm{H}$ bond scission (with an activation energy barrier of $103.1 \mathrm{~kJ}$. $\mathrm{mol}^{-1}$ ) requires less energy than $\mathrm{C}-\mathrm{O}$ bond scission (with an activation energy barrier of $249.3 \mathrm{~kJ} \cdot \mathrm{mol}^{-1}$ ). The major reaction pathway on $\mathrm{Pd}(111)$ surfaces involves $\mathrm{O}-\mathrm{H}$ bond scission in $\mathrm{CH}_{3} \mathrm{OH}$ and then a further decomposition of the resultant methoxy intermediate to $\mathrm{CO}(\mathrm{s})$ and $\mathrm{H}(\mathrm{s})$ via sequential hydrogen abstraction from $\mathrm{CH}_{3} \mathrm{O}(\mathrm{s})$. $\mathrm{O}-\mathrm{H}$ bond scission in methanol and hydrogen abstraction from the methoxy group are possible rate-determining steps for this decomposition with activation energy barriers of 103.1 and $106.7 \mathrm{~kJ} \cdot \mathrm{mol}^{-1}$, respectively.
\end{abstract}

Key Words: Methanol decomposition; Reaction mechanism; Density functional theory; Transition state

进人 21 世纪之后, 人类面临着能源与环境两 大问题. 一方面, 随着石油和煤的开采, 其资源总量 在不断减少; 另一方面, 以汽油为燃料的汽车尾气
已经成为大气污染的主要来源 ${ }^{[1,2]}$. 鉴于这种情况, 各 国都在积极寻找来源充足并且对环境和健康危害 更小的石油替代品, 甲醇是其中很有可能代替汽油

Received: December 5, 2008; Revised: January 25, 2009; Published on Web: March 4, 2009.

*Corresponding author. Email: jchx@zjut.edu.cn; Tel: +86571-88320373.

浙江省科技厅 $(2007 \mathrm{C} 1109)$ 资助项目

C. Editorial office of Acta Physico-Chimica Sinica 
作为汽车燃料的一种新型燃料 ${ }^{[3]}$. 甲醇直接分解成 $\mathrm{CO}$ 与 $\mathrm{H}_{2}$ 的反应可用于工业废气的回收利用和新 型汽车燃料, 一直是实验研究的热点 ${ }^{[1]}$. 目前, 对该反 应研究较多的是在催化剂的开发上. 甲醇裂解所使 用的催化剂 ${ }^{[1}$ 主要分为三类: 一是铜基催化剂; 二是 贵金属催化剂(以 $\mathrm{Pd}$ 和 $\mathrm{Pt}$ 基催化剂为主); 三是镍系 催化剂. 由于贵金属催化剂有着很好的活性、选择性 和稳定性而受到国内外研究者的关注. 目前在催化 剂方面的研究已取得了一定的突破, 但仍存在一些 有待解决的问题, 尽快研制出低温高效的催化剂尤 为重要. 为此, 很有必要对甲醇裂解催化反应机理作 进一步的了解. 陈文凯等问用密度泛函理论研究了 甲醇分子在 $\mathrm{Au}(111)$ 面上的吸附行为, 结果表明, 甲 醇的解离首先是 $\mathrm{O}-\mathrm{H}$ 键断裂, 然后生成甲氧基中 间体. Wang 等 $[6,7]$ 用密度泛函理论对甲醇在 $\mathrm{Ni}(111)$ 、 $\mathrm{Ni}(100)$ 面上的解离进行了研究, 结果表明, $\mathrm{O}-\mathrm{H}$ 键 与 $\mathrm{C}-\mathrm{O}$ 键、 $\mathrm{C}-\mathrm{H}$ 键相比更容易先断裂, 甲氧基上氢 的脱离是整个反应的速控步骤. Greeley 等 ${ }^{[8,9]}$ 用密度 泛函理论研究了甲醇在 $\mathrm{Cu}(111) 、 \operatorname{Pt}(111)$ 面上的解 离, 认为在 $\mathrm{Cu}(111)$ 面上, 甲氧基脱氢是整个反应的 速控步骤; 认为在 $\operatorname{Pt}(111)$ 面上, 羟基氢的脱离是整 个反应的速控步骤. 到目前为止, $\mathrm{Pd}$ 作为甲醇裂解 催化剂因其受毒物和热影响较小而引起广泛关注, 并且已有很多的相关实验研究报道. Davis 等 ${ }^{[10]}$ 研究 了甲醇在 $\operatorname{Pd}(111)$ 面上的分解反应, 推测该反应是 通过甲氧基中间体然后逐步脱氢的过程. Rebholz 等 ${ }^{[11,12]}$ 用实验方法研究了 $\mathrm{Pd}(111)$ 单晶表面上甲醇分 解反应, 通过静态二次离子质谱(SSIMS)、X 射线光 电子光谱(XPS)、程序升温脱附(TPD) 和脉冲场解析 质谱(PFDMS)检测到的吸附中间体和产物来推测甲 醇分解成 $\mathrm{CO}$ 和 $\mathrm{H}_{2}$ 要经过被吸附的甲氧基的逐步 脱氢的过程. Cabilla 等 ${ }^{[13]}$ 用 FT-IR 研究了甲醇在洁 净或 $\mathrm{Ca}$ 改性的 $\mathrm{Pd} / \mathrm{SiO}_{2}$ 上的吸附和反应, 得出该反 应历经 $\mathrm{O}-\mathrm{H}$ 键与 $\mathrm{C}-\mathrm{O}$ 键断裂两种途径. 尽管人们
对 Pd 催化甲醇裂解反应进行了大量的实验研究, 但其理论研究报道较少. Zhang 等 ${ }^{[14]}$ 用密度泛函理 论针对甲醇在 Pd 催化剂表面上第一步到底是先发 生 $\mathrm{C}-\mathrm{O}$ 键断裂还是先发生 $\mathrm{O}-\mathrm{H}$ 键断裂进行了研 究, 得出甲醇第一步先发生 $\mathrm{O}-\mathrm{H}$ 键断裂更容易. 为 了了解整个分解反应的具体过程, 我们在前人的基 础上, 构建了 $\operatorname{Pd}(111)$ 表面的周期性模型, 采用密度 泛函理论方法, 从分子水平上对整个裂解反应历程 进行模拟, 为寻找新型高效的催化剂提供理论依据.

\section{1 计算模型与方法}

\section{1 模型建立}

在计算中选取三层平板厚度和 $(3 \times 3)$ 超晶胞模 型来模拟 $\operatorname{Pd}(111)$ 表面, 相邻两层平板间的真空层厚 度为 $1 \mathrm{~nm}$, 以确保平板中的分子间相互作用足够 小. $\operatorname{Pd}(111)$ 表面的吸附位置一般有四种: 顶位(top)、 桥位(bridge)、六角密积(fcc)和面心立方(hcp)(如图 1 所示). 在计算模型中, 每一层有 9 个 Pd 原子.

\section{2 模拟方法}

全部计算使用 Accelrys 公司的 Materials Studio 4.1 软件中的 $\mathrm{DMol}^{3}$ 模块 ${ }^{[15,16]}$. 计算中对内层电子作 冻芯处理, 采用 $\mathrm{ECP}$ 赝势 ${ }^{[5]}$; 价电子波函数采用双数 值基组加极化函数展开(DNP) ${ }^{[5]}$; 交换相关势采用广 义梯度近似(GGA)和 Perdew-Wang-91 泛函相结合 的方法 $(\mathrm{GGA}-\mathrm{PW} 91)^{[17,18]}$; 计算过程中使用 $3 \times 3 \times 1$ 的 $K$ 点设置, 优化收玫精度取程序内定值; 构型优化时 底物没有固定. 据文献[19]报道, 自旋极化对吸附构 型影响很小, 所以在构型优化时不考虑自旋极化. 但 是为了解决不考虑自旋极化带来吸附能高估的问 题, 在计算能量的时候考虑了自旋极化. 过渡态计算 使用完全线性同步和二次同步变换(complete LST/ QST)方法 ${ }^{[20]}$.

采用上述方法对自由 $\mathrm{CH}_{3} \mathrm{OH}$ 分子进行几何构 型优化, 计算得到的自由 $\mathrm{CH}_{3} \mathrm{OH}$ 分子的几何构型

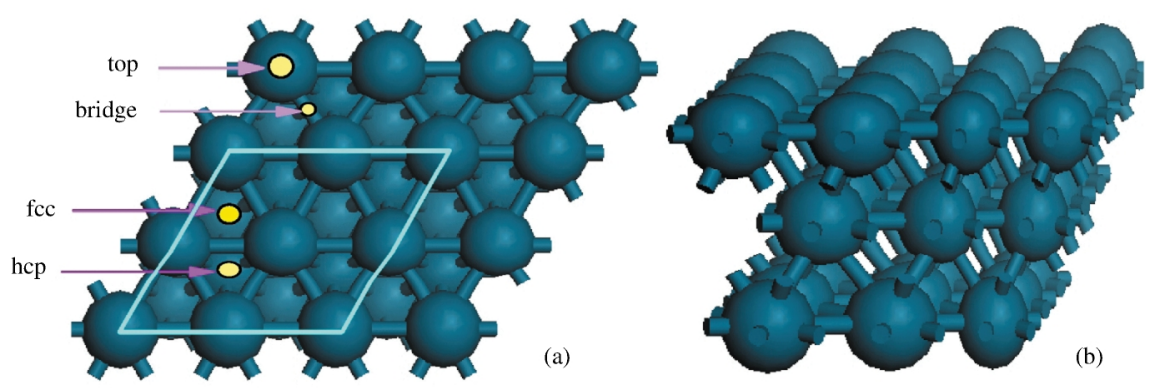

图 $1 \operatorname{Pd}(111)$ 表面的顶视图(a)和侧视图(b)

Fig.1 Top view (a) and side view (b) of $\operatorname{Pd(111)~surface~}$ 
表 1 甲醇在 $\operatorname{Pd}(111)$ 表面上裂解后各物种的几何构型参数及吸附能

Table 1 Geometric parameters and adsorption energies for species during methanol decomposition on Pd(111)

\begin{tabular}{cccccccc}
\hline Species & \multirow{2}{*}{ Preferred site } & $\begin{array}{c}\text { Atom(A) bonded } \\
\text { to Pd }\end{array}$ & $R(\mathrm{Pd}-\mathrm{A}) / \mathrm{nm}$ & $R(\mathrm{O}-\mathrm{H}) / \mathrm{nm}$ & $R(\mathrm{C}-\mathrm{O}) / \mathrm{nm}$ & $R(\mathrm{C}-\mathrm{H}) / \mathrm{nm}$ & $E_{\text {add }} /\left(\mathrm{kJ} \cdot \mathrm{mol}^{-1}\right)$ \\
\hline free- $\mathrm{CH}_{3} \mathrm{OH}$ & - & - & - & 0.0965 & 0.1424 & 0.1098 & - \\
& & & & $(0.0945)$ & $(0.1425)$ & $(0.1094)$ & \\
$\mathrm{CH}_{3} \mathrm{OH}$ & top & oxygen & 0.2425 & 0.0976 & 0.1448 & 0.1100 & 46.1 \\
$\mathrm{CH}_{3} \mathrm{O}$ & fcc & $\begin{array}{c}\text { oxygen } \\
\text { carbon and }\end{array}$ & 0.2207 & - & 0.1428 & 0.1099 & 192.5 \\
$\mathrm{CH}_{2} \mathrm{O}$ & top-bridge-top & oxygen & 0.2170 & - & 0.1307 & 0.1108 & 83.4 \\
$\mathrm{CHO}$ & top & carbon & 0.1954 & - & 0.1218 & 0.1111 & 251.5 \\
$\mathrm{CO}$ & fcc & carbon & 0.2102 & - & 0.1186 & - & 208.7 \\
$\mathrm{H}$ & fcc & hydrogen & 0.1812 & - & - & - & 318.3 \\
$\mathrm{CH}$ & top & carbon & 0.2060 & - & - & 0.1096 & 223.1 \\
$\mathrm{OH}$ & fcc & oxygen & 0.2206 & 0.0972 & - & - & 262.7 \\
\hline
\end{tabular}

The experimental values from Ref.[21] are given in parentheses.

与实验值 ${ }^{[2]}$ 较为吻合(如表 1 所示), 说明本文采用的 方法和模型是可靠的.

吸附能 $E_{\text {ads }}$ 定义为吸附前后各物质总能量的变 化, 它表示发生吸附的可能性和吸附的强弱程度. 计 算公式: $E_{\text {ads }}=E_{\text {adsorbate }}+E_{\text {substrate }}-E_{\text {system. }}$. 其中 $E_{\text {adsorbate }}$ 和 $E_{\text {substrate }}$ 分别表示吸附前吸附物和底物的能量, $E_{\text {system }}$ 表示吸附后体系的总能量.

\section{2 结果与讨论}

\section{1 各物种在金属表面的吸附}

通过对甲醇裂解过程中可能涉及到的吸附物种
进行构型优化, 计算得到的各物种在 Pd 金属表面 的最稳定吸附构型数据如表 1 所示, 最稳定吸附构 型如图 2 所示.

$\mathrm{CH}_{3} \mathrm{OH}$ 吸附: $\mathrm{CH}_{3} \mathrm{OH}$ 在 $\mathrm{Pd}(111)$ 表面最稳定的 吸附位是在顶位(图 2(a)). Grobman 等 ${ }^{[22]}$ 用 UPS(UV photoemission spectroscopy) 观测到在温度为 $120 \mathrm{~K}$ 时, 甲醇通过 $\mathrm{O}$ 原子吸附在 $\mathrm{Pd}$ 金属表面上.我们 通过计算同样发现，甲醇通过 $\mathrm{O}$ 原子与金属表面 成键, 其 $\mathrm{O}-\mathrm{Pd}$ 的距离为 $0.2425 \mathrm{~nm}$. 吸附在 $\mathrm{Pd}$ 表 面的甲醇分子构型较自由甲醇分子变化不大, 催化 剂表面吸附的甲醇分子中 $\mathrm{O}-\mathrm{H}$ 键长为 $0.0976 \mathrm{~nm}$,

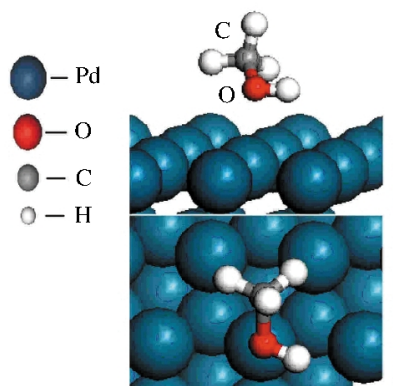

(a) $\mathrm{CH}_{3} \mathrm{OH}($ top $)$

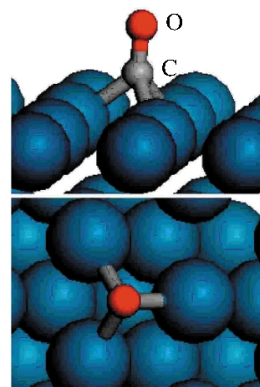

(e) $\mathrm{CO}(\mathrm{fcc})$

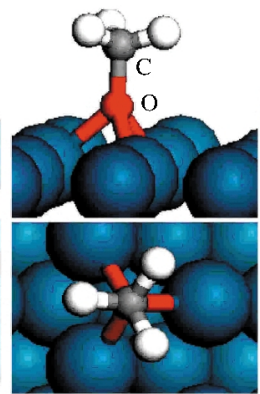

(b) $\mathrm{CH}_{3} \mathrm{O}(\mathrm{fcc})$

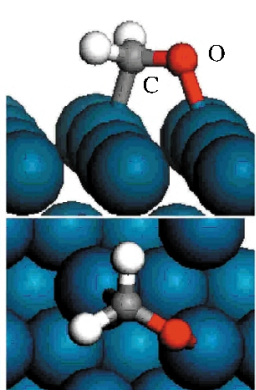

(c) $\mathrm{CH}_{2} \mathrm{O}$ (top-bridge-top)

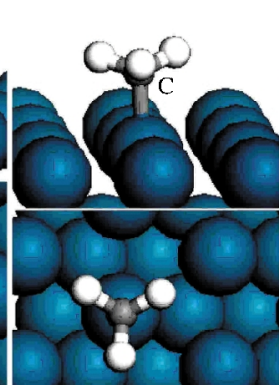

(g) $\mathrm{CH}_{3}$ (top)

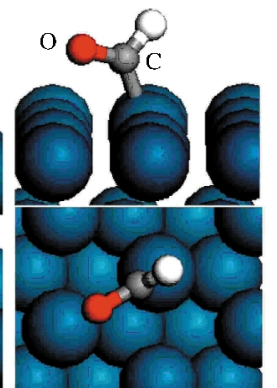

(d) $\mathrm{CHO}($ top)

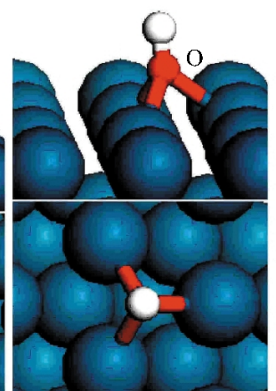

(h) $\mathrm{OH}(\mathrm{fec})$

图 2 甲醇在 $\operatorname{Pd}(\mathbf{1 1 1})$ 面上裂解后各物种的最稳定吸附构型

Fig.2 Most stable adsorption configurations of species during methanol decomposition on $\operatorname{Pd(111)~}$ Top and bottom panels for each pair of images provide a side view and a top view of the slabs, respectively. 
与自由甲醇分子中 $\mathrm{O}$ - $\mathrm{H}$ 键长 $(0.0965 \mathrm{~nm})$ 相比拉 长了 $0.0011 \mathrm{~nm}, \mathrm{C}$ - $\mathrm{O}$ 键长也伸长了 $0.0024 \mathrm{~nm}$, 而 $\mathrm{C}-\mathrm{H}$ 键长变化不大. 这说明甲醇在与钯相互作用 中削弱了 $\mathrm{O}-\mathrm{H}$ 键和 $\mathrm{C}-\mathrm{O}$ 键, 也就是说, 在甲醇靠 近钯催化剂的过程中, $\mathrm{O}-\mathrm{H}$ 键和 $\mathrm{C}-\mathrm{O}$ 键都被活化 了. 甲醇分子在该位置上的吸附能仅为 $46.1 \mathrm{~kJ} \cdot \mathrm{mol}^{-1}$. Shustorovich 等 ${ }^{[23]}$ 用 BOC 方法计算得到的吸附能为 $50.2 \mathrm{~kJ} \cdot \mathrm{mol}^{-1}$. Zhang 等 ${ }^{[14]}$ 用 DFT-GGA 方法计算的 吸附能为 $35.7 \mathrm{~kJ} \cdot \mathrm{mol}^{-1}$. 这些结果都表明, 甲醇与该 催化剂表面的相互作用是十分微弱的.

$\mathrm{CH}_{3} \mathrm{O}$ 吸附: Chen 等 ${ }^{[24]}$ 通过 XPS 和 SIMS 观测 到在相应的温度下, $\operatorname{Pd}(111)$ 表面有该物种的存在, 它很容易通过 $\mathrm{CH}_{3} \mathrm{OH}$ 上的 $\mathrm{O}-\mathrm{H}$ 键断裂而形成. 由此我们推测, 甲醇裂解过程可能是经历该中间体 的过程. 计算结果发现, $\mathrm{CH}_{3} \mathrm{O}$ 在 $\mathrm{Pd}(111)$ 表面最稳 定的吸附位是在 fcc 位(图 2(b)), 通过 $\mathrm{O}$ 原子与金 属表面成键, 且 $\mathrm{C}-\mathrm{O}$ 键轴垂直于表面, 其 $\mathrm{O}$ 原子 与 $\mathrm{Pd}$ 原子间的距离为 $0.2207 \mathrm{~nm}$, 吸附能为 192.5 $\mathrm{kJ} \cdot \mathrm{mol}^{-1}$, 比 $\mathrm{CH}_{3} \mathrm{OH}$ 的吸附强很多, 这也表明它在 该金属表面不容易脱附, 在一定的温度下有进一步 发生解离的趋势.

$\mathrm{CH}_{2} \mathrm{O}$ 吸附: $\mathrm{CH}_{2} \mathrm{O}$ 在 $\mathrm{Pd}(111)$ 表面最稳定的吸 附位是在 top-bridge-top 位(图 2(c)), 其吸附能为83.4 $\mathrm{kJ} \cdot \mathrm{mol}^{-1}$. C 原子和 $\mathrm{O}$ 原子均与金属表面成键, 其 $\mathrm{C}-\mathrm{Pd}$ 的距离为 $0.2170 \mathrm{~nm}, \mathrm{O}-\mathrm{Pd}$ 的距离为 0.2120 $\mathrm{nm}$, 羰基官能团几乎是与金属表面平行的. 与 Davis 等[25,26]所报道的结论是一致的.

CHO 吸附: 文献[27-29]曾报道在各种过渡金属 表面上, $\mathrm{CHO}$ 是甲醇裂解的中间体之一. 通过计算发 现, CHO 在 $\mathrm{Pd}(111)$ 表面最稳定的吸附位是在顶位 (图 2(d)), 它通过 C 原子与金属表面成键, 其 C-Pd 的距离为 $0.1954 \mathrm{~nm}$, 吸附能为 $251.5 \mathrm{~kJ} \cdot \mathrm{mol}^{-1}$. CHO 在 $\mathrm{Ni}(111)^{[6]}$ 和 $\mathrm{Pt}(111)^{[9]}$ 的吸附能分别为 232.0 和 $227.5 \mathrm{~kJ} \cdot \mathrm{mol}^{-1}$. 可见, 该中间体与过渡金属表面有着 强相互作用.

$\mathrm{CO}$ 吸附: $\mathrm{CO}$ 在 $\mathrm{Pd}(111)$ 表面最稳定的吸附位 是在 fcc 位(图 2(e)), 通过 C 原子与金属表面成键, 且 $\mathrm{C}$ - $\mathrm{O}$ 键轴垂直于表面, 其 $\mathrm{C}$ 原子与 $\mathrm{Pd}$ 原子间的 距离为 $0.2102 \mathrm{~nm}$, 吸附能为 $208.7 \mathrm{~kJ} \cdot \mathrm{mol}^{-1}$. 魏子 栋等 ${ }^{[30]}$ 用 DFT 方法, 选用族模型计算得到了 $\mathrm{CO}$ 在 $\operatorname{Pd}(111)$ 面上最稳定的吸附位是桥位, 其吸附能为 $205.3 \mathrm{~kJ} \cdot \mathrm{mol}^{-1}$. 我们的计算结果与之不一样, 这可能 跟选用的计算模型不同有关.
$\mathrm{H}$ 吸附: $\mathrm{H}$ 在 $\mathrm{Pd}(111)$ 表面最稳定的吸附位是在 $\mathrm{fcc}$ 位(图 2(f)), 它通过 $\mathrm{H}$ 原子与金属表面成键, 其 $\mathrm{H}$ 原子与 $\mathrm{Pd}$ 原子间的距离为 $0.1812 \mathrm{~nm}$, 远小于 $\mathrm{H}$ 与 $P d$ 原子的范德华半径之和 $(0.2830 \mathrm{~nm})^{[31]}$, 其吸附 能高达 $318.3 \mathrm{~kJ} \cdot \mathrm{mol}^{-1}$, 说明 $\mathrm{H}$ 与 $\mathrm{Pd}$ 原子之间形成 化学吸附. $\mathrm{H}$ 在 $\mathrm{Ni}(111)^{[6]} 、 \mathrm{Cu}(111)^{[8]}$ 和 $\mathrm{Pt}(111)^{[9]}$ 表面 上最稳定的吸附位均在 fcc 位, 且吸附能均大于 200 $\mathrm{kJ} \cdot \mathrm{mol}^{-1}$, 表现出很强的化学吸附能力.

$\mathrm{CH}_{3} 、 \mathrm{OH}$ 吸附: Rebholz 等 ${ }^{[1]}$ 曾通过 SSIMS 和 XPS 观测到在一定温度范围内有这两个物种的存 在. 于是, 我们对此进行计算并发现, $\mathrm{CH}_{3}$ 在 $\mathrm{Pd}(111)$ 表面最稳定的吸附位是在顶位(图 2(g)), 它平行于 金属表面, 通过 C 原子与金属表面成键, 其 C 原子 与 $\mathrm{Pd}$ 原子间的距离为 $0.2060 \mathrm{~nm}$, 吸附能为 223.1 $\mathrm{kJ} \cdot \mathrm{mol}^{-1}$. OH 在 $\mathrm{Pd}(111)$ 表面最稳定的吸附位是在 fcc 位(图 2(h)), O- $-\mathrm{H}$ 键轴垂直于金属表面, 通过 $\mathrm{O}$ 原子与金属表面成键, 其 $\mathrm{O}$ 原子与 $\mathrm{Pd}$ 原子间的距 离为 $0.2206 \mathrm{~nm}$, 吸附能为 $262.7 \mathrm{~kJ} \cdot \mathrm{mol}^{-1}$.

\section{2 反应历程}

\subsection{1 $\mathrm{CH}_{3} \mathrm{OH}(\mathrm{s}) \rightarrow \mathrm{CH}_{3} \mathrm{O}(\mathrm{s})+\mathrm{H}(\mathrm{s})$}

对于 $\mathrm{CH}_{3} \mathrm{OH}$ 在 $\mathrm{Pd}(111)$ 上脱羟基 $(\mathrm{OH})$ 氢的过 程如图 3 所示. 以 $\mathrm{CH}_{3} \mathrm{OH}$ 吸附在顶位作为反应初 始态, 在各个位置吸附状态中, 该状态体系总能最 低. 此时, $\mathrm{O}-\mathrm{H}$ 键的键长为 $0.0976 \mathrm{~nm}$. 以 $\mathrm{CH}_{3} \mathrm{O}$ 吸 附在 Pd-fcc 位, $\mathrm{H}$ 吸附在最邻近的 Pd-fcc 位的共吸 附体系作为反应终态, 该体系总能最低, 然后进行过 渡态搜索. 结果得到该过程的活化能为 $103.1 \mathrm{~kJ}$. $\mathrm{mol}^{-1}$, 反应前后能量相差 $50.5 \mathrm{~kJ} \cdot \mathrm{mol}^{-1}$, 为吸热反 应. 我们计算的活化能值比 Zhang 等 ${ }^{[14]}$ 的计算结果 $\left(78.0 \mathrm{~kJ} \cdot \mathrm{mol}^{-1}\right)$ 稍大些, 这可能与计算精度的设置有

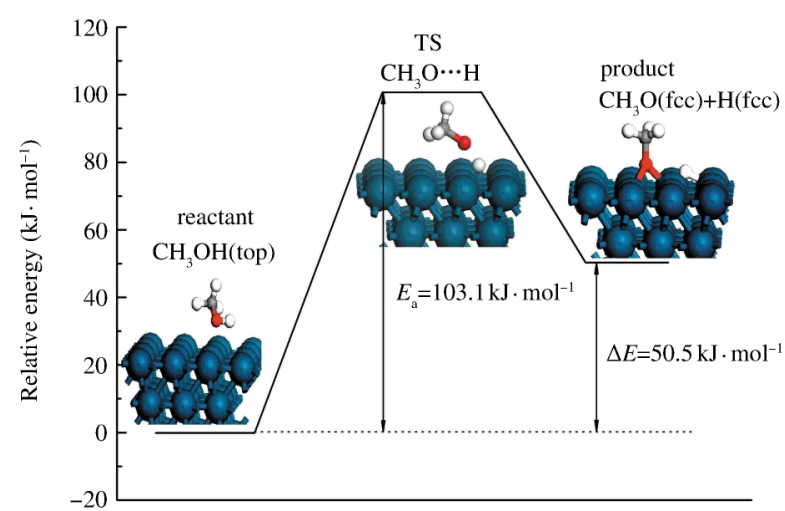

图 $3 \mathrm{CH}_{3} \mathrm{OH}$ 在 $\mathrm{Pd}(111)$ 面上脱羟基氢的能量变化示意图

Fig.3 Energy change for the abstraction of hydroxyl hydrogen from methanol on $\operatorname{Pd}(111)$

TS: transition state; $E_{\mathrm{a}}$ : activation energy 
关. 通过对过渡态的结构进行分析发现, $\mathrm{O}-\mathrm{H}$ 键的 键长由原来的 $0.0976 \mathrm{~nm}$ 拉长到 $0.2205 \mathrm{~nm}$, 这说明 $\mathrm{CH}_{3} \mathrm{OH}$ 上的 $\mathrm{O}-\mathrm{H}$ 键的成键作用已经很小, 此时的 $\mathrm{H}$ 原子可以认为脱离了 $\mathrm{O}$ 原子. 同时, $\mathrm{O}-\mathrm{H}$ 键中的 $\mathrm{H}$ 原子与 $\mathrm{Pd}$ 原子间的距离由原先的 $0.3043 \mathrm{~nm}$ 缩 短到现在的 $0.1719 \mathrm{~nm}, \mathrm{O}$ 原子与 $\mathrm{Pd}$ 原子间的距离 由 $0.2425 \mathrm{~nm}$ 缩短到 $0.2032 \mathrm{~nm}$, 表明 $\mathrm{O}-\mathrm{H}$ 键已经 被活化, 并最终解离吸附在 $\operatorname{Pd}(111)$ 表面.

\subsection{2 $\quad \mathrm{CH}_{3} \mathrm{O}(\mathrm{s}) \rightarrow \mathrm{CH}_{2} \mathrm{O}(\mathrm{s})+\mathrm{H}(\mathrm{s})$}

对于 $\mathrm{CH}_{3} \mathrm{O}$ 在 $\mathrm{Pd}(111)$ 上脱氢的过程如图 4 所 示. 以 $\mathrm{CH}_{3} \mathrm{O}$ 吸附在 Pd-fcc 位作为反应初始态, 该吸 附状态体系总能最低. 此时, $\mathrm{C}$ 与解离的 $\mathrm{H}$ 距离为 $0.1099 \mathrm{~nm}$. 以 $\mathrm{CH}_{2} \mathrm{O}$ 吸附在 Pd-top-bridge-top 位, $\mathrm{H}$ 吸附在 Pd-fcc 位的共吸附体系作为反应终态, 该体 系总能最低, 然后进行过渡态搜索. 结果获得该过程 的活化能为 $106.7 \mathrm{~kJ} \cdot \mathrm{mol}^{-1}$, 反应前后能量相差 12.0 $\mathrm{kJ} \cdot \mathrm{mol}^{-1}$, 为吸热反应. 通过对过渡态的结构进行分 析发现, C 与解离的 $\mathrm{H}$ 距离由原先的 $0.1099 \mathrm{~nm}$ 拉 长到 $0.2228 \mathrm{~nm}$, 同时, 解离的这个 $\mathrm{H}$ 原子与 $\mathrm{Pd}$ 原 子间的距离由原先的 $0.3542 \mathrm{~nm}$ 缩短到现在的 $0.1679 \mathrm{~nm}, \mathrm{C}$ 原子与 $\mathrm{Pd}$ 原子间的距离由 $0.3346 \mathrm{~nm}$ 缩短到 $0.3082 \mathrm{~nm}$, 表明该 $\mathrm{C}-\mathrm{H}$ 键已经被活化, 并 最终解离吸附在 $\operatorname{Pd}(111)$ 表面.

\subsection{3 $\mathrm{CH}_{2} \mathrm{O}(\mathrm{s}) \rightarrow \mathrm{CHO}(\mathrm{s})+\mathrm{H}(\mathrm{s})$}

对于 $\mathrm{CH}_{2} \mathrm{O}$ 在 $\mathrm{Pd}(111)$ 上脱氢的过程如图 5 所 示. 以 $\mathrm{CH}_{2} \mathrm{O}$ 吸附在 Pd-top-bridge-top 位作为反应初 始态, 在各种吸附位中该吸附状态体系总能最低. 此 时, $\mathrm{C}$ 与解离的 $\mathrm{H}$ 距离为 $0.1108 \mathrm{~nm}$. 以 $\mathrm{CHO}$ 吸附 在顶位, $\mathrm{H}$ 吸附在最邻近的 Pd-fcc 位的共吸附体系 总能最低, 所以以此作为反应终态, 然后进行过渡态 搜索. 结果获得该过程的活化能为 $53.6 \mathrm{~kJ} \cdot \mathrm{mol}^{-1}$, 反

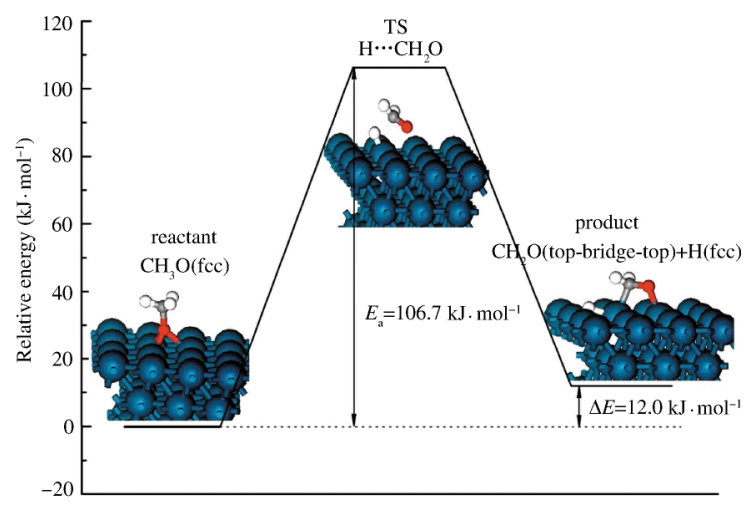

图 $4 \mathrm{CH}_{3} \mathrm{O}$ 在 $\mathrm{Pd}(111)$ 面上脱氢的能量变化示意图

Fig.4 Energy change for the abstraction of hydrogen from methoxy on $\operatorname{Pd}(111)$
应前后能量相差 $-20.6 \mathrm{~kJ} \cdot \mathrm{mol}^{-1}$, 为放热反应. 从能量 角度来考虑, 该过程较前面两个反应更容易进行. 通 过对过渡态的结构进行分析发现, C 与解离的 $\mathrm{H}$ 距 离为 $0.1526 \mathrm{~nm}$, 比初始态拉长了 $0.0418 \mathrm{~nm}$, 此时 的 $\mathrm{C}$ 原子与 $\mathrm{Pd}$ 原子的距离为 $0.2065 \mathrm{~nm}$, 解离的 $\mathrm{H}$ 原子与 $\mathrm{Pd}$ 原子的距离为 $0.1721 \mathrm{~nm}$, 均比始态 $(0.2170,0.2545 \mathrm{~nm})$ 更靠近金属表面, 并且 $\mathrm{C}$ 原子与 解离的 $\mathrm{H}$ 原子共同与同一个 $\mathrm{Pd}$ 原子成键, 从而也 说明该 C- $\mathrm{H}$ 键被活化, 并最终解离吸附在 $\mathrm{Pd}(111)$ 表面.

\subsection{4 $\mathrm{CHO}(\mathrm{s}) \rightarrow \mathrm{CO}(\mathrm{s})+\mathrm{H}(\mathrm{s})$}

对于 $\mathrm{CHO}$ 在 $\mathrm{Pd}(111)$ 上脱氢的过程如图 6 所 示. 以 $\mathrm{CHO}$ 吸附在顶位作为反应初始态, 此时, $\mathrm{C}$ 与 $\mathrm{H}$ 原子的距离为 $0.1111 \mathrm{~nm}$. 以 $\mathrm{CO}$ 吸附在 $\mathrm{Pd}-\mathrm{fcc}$ 位, $\mathrm{H}$ 吸附在最邻近的 Pd-fcc 位的共吸附体系作为 反应终态, 然后进行过渡态搜索. 结果获得该过程的 活化能为 $70.7 \mathrm{~kJ} \cdot \mathrm{mol}^{-1}$, 反应前后能量相差-107.9 $\mathrm{kJ} \cdot \mathrm{mol}^{-1}$, 为放热反应. 通过对过渡态的结构进行分 析发现, $\mathrm{C}$ 与 $\mathrm{H}$ 原子的距离为 $0.1402 \mathrm{~nm}$, 比初始态 拉长了 $0.0291 \mathrm{~nm}$, 此时 $\mathrm{C}$ 原子与 $\mathrm{Pd}$ 原子的距离为 $0.1947 \mathrm{~nm}$, 与始态 $(0.1954 \mathrm{~nm})$ 变化不大, 而 $\mathrm{H}$ 原子 与 $\mathrm{Pd}$ 原子的距离为 $0.1807 \mathrm{~nm}$, 比始态 $(0.2628 \mathrm{~nm})$ 更靠近金属表面, 此时 $\mathrm{C}$ 原子与 $\mathrm{H}$ 原子共同与同 一个 $\mathrm{Pd}$ 原子成键, 从而也说明该 $\mathrm{C}-\mathrm{H}$ 键被活化, 并最终解离吸附在 $\operatorname{Pd}(111)$ 表面. 另外, 我们发现在 过渡态中, $\mathrm{C}-\mathrm{O}$ 键键长为 $0.1179 \mathrm{~nm}$, 而始态时 $\mathrm{C}-\mathrm{O}$ 键键长为 $0.1218 \mathrm{~nm}, \mathrm{C}-\mathrm{O}$ 键键长缩短了 0.0039 $\mathrm{nm}$, 说明该过程使 $\mathrm{C}$ - $\mathrm{O}$ 键进一步加强, 从而也说明 $\mathrm{CO}$ 继续分解的难度增大.

\section{$2.2 .52 \mathrm{H}(\mathrm{s}) \rightarrow \mathrm{H}_{2}(\mathrm{~g})$}

首先对两个 $\mathrm{H}(\mathrm{s})$ 在 $\mathrm{Pd}(111)$ 面共吸附的构型进

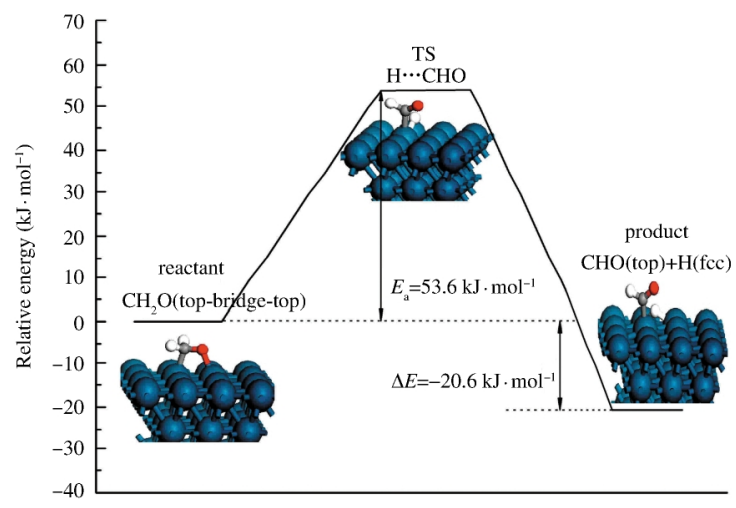

图 $5 \mathrm{CH}_{2} \mathrm{O}$ 在 $\mathrm{Pd}(111)$ 面上脱氢的能量变化示意图

Fig.5 Energy change for the abstraction of hydrogen from formaldehyde on Pd(111) 


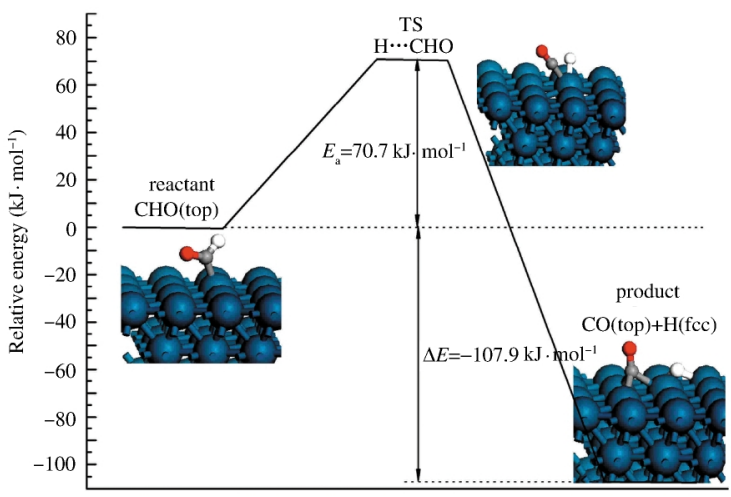

图 $6 \mathrm{CHO}$ 在 $\mathrm{Pd}(111)$ 面上脱氢的能量变化示意图

Fig.6 Energy change for the abstraction of hydrogen from formyl on $\operatorname{Pd}(111)$

行优化, 计算结果表明, 两个 $\mathrm{H}(\mathrm{s})$ 共吸附时优势吸附 位均为 fcc 位, 该吸附状态时的体系总能最小, 此时, 两个 $\mathrm{H}$ 原子之间的距离为 $0.2834 \mathrm{~nm}$, 以此作为该 步骤的反应起点, 进行过渡态搜索, 过程如图 7 所 示. 通过对过渡态分析可知, 两个 $\mathrm{H}$ 原子之间的距 离为 $0.1381 \mathrm{~nm}$, 说明该过程是两个 $\mathrm{H}(\mathrm{s})$ 首先各自 向桥位移动, 然后结合为 $\mathrm{H}_{2}$, 最后从表面脱附的过 程. 该步骤的活化能为 $89.8 \mathrm{~kJ} \cdot \mathrm{mol}^{-1}$. 反应前后能 量相差 $65.7 \mathrm{~kJ} \cdot \mathrm{mol}^{-1}$, 为吸热反应.

\subsection{6 $\quad \mathrm{CH}_{3} \mathrm{OH}(\mathrm{s}) \rightarrow \mathrm{CH}_{3}(\mathrm{~s})+\mathrm{OH}(\mathrm{s})$}

对于 $\mathrm{CH}_{3} \mathrm{OH}$ 在 $\mathrm{Pd}(111)$ 上发生 $\mathrm{C}-\mathrm{O}$ 键断裂的 过程如图 8 所示. 以 $\mathrm{CH}_{3} \mathrm{OH}$ 吸附在 Pd-top 位作为 反应初始态, 此时, $\mathrm{C}-\mathrm{O}$ 键键长为 $0.1448 \mathrm{~nm}$. 以 $\mathrm{CH}_{3}$ 吸附在 Pd-top 位, $\mathrm{OH}$ 吸附在 Pd-fcc 位的共吸 附体系作为反应终态, 然后进行过渡态搜索. 结果 获得该过程的活化能为 $249.3 \mathrm{~kJ} \cdot \mathrm{mol}^{-1}$, 反应前后

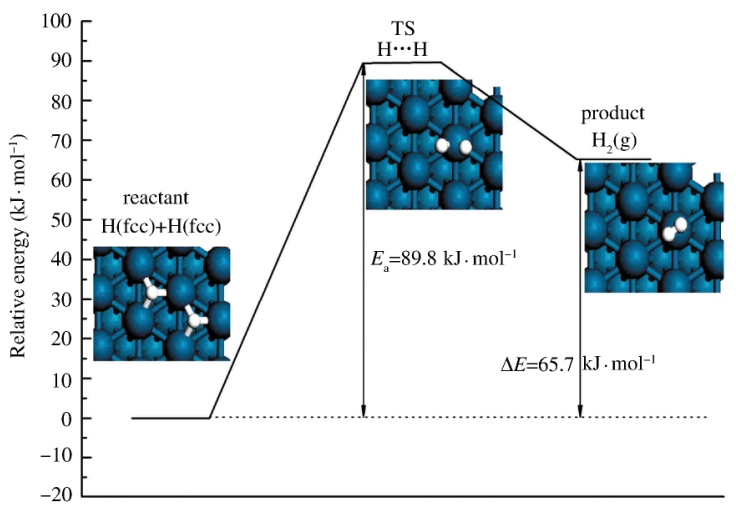

图 $7 \mathrm{H}_{2}$ 从 $\operatorname{Pd}(111)$ 面上脱附的能量变化示意图 Fig.7 Energy change for $\mathrm{H}_{2}$ recombinative desorption on $\operatorname{Pd}(111)$

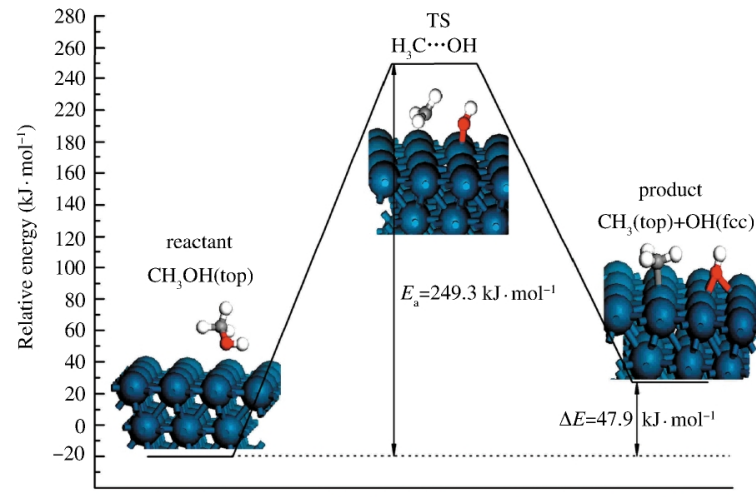

图 $8 \mathrm{CH}_{3} \mathrm{OH}$ 在 $\mathrm{Pd}(111)$ 面上发生 $\mathrm{C}-\mathrm{O}$ 键断裂的能量 变化示意图

Fig.8 Energy change for $\mathrm{C}-\mathrm{O}$ bond scission from methanol on $\operatorname{Pd}(111)$

能量相差 $47.9 \mathrm{~kJ} \cdot \mathrm{mol}^{-1}$, 为吸热反应. Zhang 等 ${ }^{[14]}$ 计 算的活化能为 $171.6 \mathrm{~kJ} \cdot \mathrm{mol}^{-1}$, 反应前后能量相差 $13.5 \mathrm{~kJ} \cdot \mathrm{mol}^{-1}$. 通过对过渡态的结构进行分析发现, $\mathrm{C}$ 与 $\mathrm{O}$ 原子的距离为 $0.2411 \mathrm{~nm}$, 比始态拉长了 $0.0963 \mathrm{~nm}$, 可以认为 $\mathrm{C}-\mathrm{O}$ 键已经发生了断裂. 此 时 $\mathrm{O}$ 原子与 $\mathrm{Pd}$ 原子间的距离已经从始态的 0.2425 $\mathrm{nm}$ 缩短到 $0.2146 \mathrm{~nm}$, 说明它与金属表面的相互作 用进一步加强. 从活化能数据来看, $\mathrm{C}$ - $\mathrm{O}$ 键发生断 裂的难度比 $\mathrm{O}-\mathrm{H}$ 键发生断裂要大得多.

\section{3 结 论}

采用基于密度泛函理论的量子力学计算方法, 通过计算模拟从理论上对 Pd 催化甲醇裂解的反应 机理进行了研究. 通过对各物种的吸附构型进行几 何优化以及对各个基元反应进行过渡态搜索, 获得 了反应路径上各物种的吸附能和各基元反应的活化 能数据. 计算结果表明, 在 $\operatorname{Pd}(111)$ 表面上, 甲醇是所 有吸附物种中吸附最弱的 (吸附能仅为 $46.1 \mathrm{~kJ} \cdot \mathrm{mol}^{-1}$ ); 甲醇在裂解过程中, 发生 $\mathrm{O}-\mathrm{H}$ 键断裂与发生 $\mathrm{C}-\mathrm{O}$ 键断裂相比, 前者所需要的活化能为 $103.1 \mathrm{~kJ} \cdot \mathrm{mol}^{-1}$, 而后者需要的活化能很高 $\left(249.3 \mathrm{~kJ} \cdot \mathrm{mol}^{-1}\right)$, 因此甲 醇裂解反应更容易先发生 $\mathrm{O}-\mathrm{H}$ 键的断裂; 甲醇裂 解过程主要历经 $\mathrm{O}-\mathrm{H}$ 键的断裂, 生成甲氧基中间 体, 然后甲氧基中间体再逐步脱氢生成 $\mathrm{CO}(\mathrm{s})$ 和 $\mathrm{H}$ (s), 最后 $\mathrm{CO}$ 和 $\mathrm{H}_{2}$ 从金属表面脱附出来, 其中甲氧 基上脱氢的活化能为 $106.7 \mathrm{~kJ} \cdot \mathrm{mol}^{-1}$, 与甲醇发生 $\mathrm{O}-\mathrm{H}$ 键断裂的活化能差异并不大, 均有可能是整个 裂解反应的速控步骤. 


\section{References}

1 Sun, K. B.; Wang, B. M. Science Techology Information Development and Economy, 2005, 15(13): 148 [孙康波, 王保民. 科技情报开 发与经济, 2005, 15(13): 148]

2 Hellen, H.; Hakola, H.; Laurila, T. Atmos Environ., 2003, 37(11): 1413

3 Peng, B. X.; Gan, C. S.; Yan, T. T. Prog. Chem., 2004, 16(3): 414 [彭必先, 甘昌胜, 间天堂. 化学进展, 2004, 16(3): 414]

4 Zhou, W. J.; Li, W. Z.; Zhou, Z. H.; Song, S. Q.; Wei, Z. B.; Sun, G. Q.; Tsiakaras, P.; Xin, Q. Chem. J. Chin. Univ., 2003, 24: 858 [周卫江, 李文震, 周振华, 宋树芹, 魏昭涁, 孙公权, Tsiakaras, P., 辛 勤. 高等学校化学学报, 2003, 24: 858]

5 Liu, S. H.; Chen, W. K.; Cao, M. J.; Xu, Y.; Li, J. Q. Chin. J. Catal., 2006, 27(1): 55 [刘书红, 陈文凯, 曹梅娟, 许 莹, 李俊钱. 催化学报, 2006, 27(1): 55]

6 Wang, G. C.; Zhou, Y. H.; Morikawa, Y.; Nakamura, J.; Cai, Z. S.; Zhao, X. Z. J. Phys. Chem. B, 2005, 109(25): 12431

7 Zhou, Y. H.; Lv, P. H.; Wang, G. C. J. Mol. Catal. A, 2006, 258 (1-2): 203

8 Greeley, J.; Mavrikakis, M. J. Catal., 2002, 208(2): 291

9 Greeley, J.; Mavrikakis, M. J. Am. Chem. Soc., 2002, 124: 7193

10 Davis, J. L.; Barteau, M. A. Surf. Sci., 1987, 187: 387

11 Rebholz, M.; Kruse, N. J. Chem. Phys., 1991, 95(10): 7745

12 Kruse, N.; Rebholz, M.; Matolin, V.; Chuah, G. K.; Block, J. H. Surf. Sci., 1990, 238: L457

13 Cabilla, G. C.; Bonivardi, A. L.; Baltanas, M. A. J. Catal., 2001, 201(2): 213

14 Zhang, C. J.; Hu, P. J. Chem. Phys., 2001, 115(15): 7182

15 Delley, B. J. Chem. Phys., 1990, 92(1): 508

16 Delley, B. J. Chem. Phys., 2000, 113(18): 7756

17 Perdew, J. P.; Chevary, J. A.; Vosko, S. H.; Jackson, K. A.;
Pederson, M. R.; Singh, D. J.; Fiolhais, C. Phys. Rev. B, 1992, 46 (11): 6671

18 White, J. A.; Bird, D. M.; Payne, M. C.; Stich, I. Phys. Rev. Lett., 1994, 73(10): 1404

19 Ge, Q.; Jenkins, S. J.; King, D. A. Chem. Phys. Lett., 2000, 327(34): 125

20 Zhang, X. H.; Zhang, M. H.; Jiang, H. X. J. Mol. Catal.(China), 2006, 20(6): 535 [张霄航, 张敏华, 姜浩锡. 分子催化, 2006, 20(6): 535]

21 Lide, D. R. CRC handbook of chemistry and physics. 81th ed. BocaRaton: CRC Press, 2000, Chapter 9: 36

22 Luth, H.; Rubloff, G. W.; Grobman, W. D. Surf. Sci., 1977, 63: 325

23 Shustorovich, E.; Bell, A. T. J. Catal., 1988, 113: 341

24 Chen, J. J.; Jiang, Z. C.; Zhou, Y.; Chakraborty, B. R.; Winograd, N. Surf. Sci., 1995, 328: 248

25 Davis, J. L.; Barteau, M. A. J. Am. Chem. Soc., 1989, 111: 1782

26 Davis, J. L.; Barteau, M. A. Surf. Sci., 1990, 235: 235

27 Yang, H. Surf. Sci., 1995, 343: 61

28 Gomes, J. R. B.; Gomes, J. A. N. F. J. Electroanal. Chem., 2000, 483: 180

29 Haq, S.; Love, J. G.; Sansders, H. E.; King, D. A. Surf. Sci., 1995, 325: 230

30 Yan, A. Z.; Wei, Z. D.; Li, L. L.; Zhang, F. Q. J. Chongqing Univ., 2007, 30(6): 48 [闵爱芝, 魏子栋, 李兰兰, 张凤桥. 重庆大学学 报, 2007, 30(6): 48]

31 Mai, S. W.; Zhou, G. D.; Li, W. J. Advanced inorganic structure chemistry. Beijing: Peking University Press, 2001: 101 [麦松威, 周公度, 李伟基. 高等无机结构化学. 北京: 北京大学出版社, 2001: 101] 